\title{
AJRC
}

\author{
Australia - Japan Research Centre
}

\section{Asymmetric Increasing Trends in Dependence in International Equity Markets}

\section{AJRC Working Paper 05/2014 July 2014}

Tatsuyoshi Okimoto*

Australia-Japan Research Centre

Australian National University

\section{Abstract}

This paper investigates asymmetric increasing trends in dependence in major international equity markets. To this end, we develop a multiple-regime smooth-transition copula GARCH model and address several important questions, including the number of regimes and the existence of increasing asymmetric trends in dependence. Our results suggest that two or three regimes are sufficient for describing the dependence trends in international equity markets over the last 35 years with significant asymmetric increases. In addition, the implied time-series of three dependence measures show a wide variety of dynamics, demonstrating the usefulness of our framework to describe asymmetric increasing dependence trends. Finally, we evaluate the economic significance of our empirical finding based on the $99 \%$ value at risk and expected shortfall. Our result indicates that both risk measures have increased approximately $20 \%$ over the last 35 years in major equity markets. 
*Associate Professor, Crawford School of Public Policy, Australian National University, Crawford Building \#132, Lennox Crossing, Canberra ACT 0200, AUSTRALIA. Phone: +61-2-6125-4763. Fax: +61-2-61258448. E-mail: tatsuyoshi.okimoto@anu.edu.au.

\section{Keywords}

Smooth transition model; Copula; Spearman's rho; Tail dependence

\section{JEL Classification}

C32; C51; G15

\section{Suggested Citation}

Okimoto, T., 2014. Asymmetric Increasing Trends in Dependence in International Equity Markets, AJRC Working Paper No.5/2014, June. Australia-Japan Research Centre, Crawford School of Public Policy, The Australian National University.

\section{Address for correspondence}

(E) ajrc@anu.edu.au

ISSN 07288409

ISBN 978-0-86413-005-1

The Australia-Japan Research Centre (AJRC) conducts research to explore and improve understanding of the economies and economic policy processes in Australia and Japan and both countries' strategic interests in the Asia Pacific economy.

The AJRC Working Paper Series aims to provide a forum for the exchange of topical research and contains the latest research and analysis on the Japanese economy, as well as the political economy, regional integration and trade. The views expressed in AJRC Working Papers are those of the individual authors and do not represent the views of the Australia-Japan Research Centre, the Crawford School, or the institutions to which authors are attached.

The Crawford School of Public Policy is the Australian National University's public policy school, serving and influencing Australia, Asia and the Pacific through advanced policy research, graduate and executive education, and policy impact. 


\section{Introduction}

The study of time-varying dependence structures in international equity markets has recently attracted increasing attention among theorists, empirical researchers, and practitioners for numerous reasons. For instance, investors need to assess the degree of comovement among international stock returns accurately to construct a well diversified portfolio. In addition, to evaluate risk measures, such as the Value at Risk (VaR) and expected shortfall (ES), risk managers should take into account interdependence in international equity markets. Ignoring an increase in dependence could lead to a considerable under-evaluation of those risk measures. Policy makers also have to pay close attention to contagion, which is caused by dependence between extreme negative shocks across international financial markets. If contagion effects became important, a financial crisis occurring in one country would have substantial effects on other countries, amplifying concerns for policy makers as well as market investors.

Another reason for the existence of a growing number of studies on dynamics of dependence in international equity markets is associated with financial market integration. Over the last three decades, the circumstances of the world financial markets have changed dramatically. Examples of this include an increase of world economic relations, competition and globalization, development of the world transportation system, reduction of trade barriers, and evolution in information technology. In addition, according to the IMF's Annual Report on Exchange Arrangements and Exchange Restrictions (AREAER), ${ }^{1}$ many industrial countries have experienced a rapid increase in their degree of financial openness since the mid-1980s. One natural consequence of these changes is a promotion of financial market integration. Indeed, the de facto measures recently constructed by Lane and Milesi-Ferretti (2007) indicate that financial integration in industrial countries was promoted gradually throughout the 1970s and 1980s, and accelerated throughout the mid-1990s. ${ }^{2}$ It is not unreasonable to assume that the promotion of financial market integration would affect

\footnotetext{
${ }^{1}$ AREAER reports a set of de jure measures of restrictions on cross-border capital flows, and is widely used to measure financial openness.

${ }^{2}$ See Kose et al. (2009) for details of financial integration and related measures.
} 
comovements among international financial markets.

The main contribution of this paper is to investigate asymmetric increasing trends in dependence in international equity markets systematically. In particular, the paper addresses the following questions: (i) Is the multivariate Normal (MVN) model appropriate for modeling dependence trends in international equity markets? (ii) How many dependence regimes are sufficient to characterize the dependence trends over the last 35 years? (iii) Is there an increasing trend in dependence in international equity markets? (iv) Is there any asymmetry in the dependence trends between the upper and lower tails of the distribution? (v) When did important changes in the dependence trends occur? (vi) Which tail of the distribution contributed most to these changes?

The paper is related to voluminous previous studies investigating time-varying dependence in international equity markets. For instance, Von Furstenberg and Jeon (1989) estimate a VAR model consisting of daily stock returns for four major markets (Japan, Germany, U.K., and U.S.) and detect an increase in correlations since the crash of 1987. Koch and Koch (1991) reach a similar conclusion based on the daily returns across eight different national equity markets. On the other hand, King, Sentana, and Wadhwani (1994) claim that their finding of increasing dependence only reflected a transitory increase caused by the 1987 crash. To test an increase in correlation more precisely, Longin and Solnik (1995) estimate a bivariate GARCH model with a trend term in correlations between the US and six major countries. They find a significant increase in correlations for four out of six pairs. Berben and Jansen (2005) model the dynamics of correlation with a smooth transition model and show that correlations among the German, U.K., and U.S. stock markets have doubled, whereas Japanese correlations have remained the same. On the other hand, Bekaert, Hodrick, and Zhang (2009) establish that there is no evidence for an upward trend in international stock return correlations, except for the European stock markets based on parsimonious risk-based factor models. Finally, Christoffersen et al. (2012) examine copula correlations in international stock markets and find a significant increasing trend that can be explained by neither volatility nor other financial and macroeconomic variables. Thus, whereas there has been much empirical work in this area, it is fair to say that there is not enough evidence that 
dependence in international equity markets is permanently and asymmetrically increasing. This paper will provide another evidence of asymmetric increasing dependence in international equity markets.

Recently, Kumar and Okimoto (2011) propose the two-regime smooth transition copulaGARCH (STCG) model to examine correlation dynamics in international government securities markets. This paper extends their model to the multiple-regime smooth transition asymmetric copula model, differing from the aforementioned previous studies in several ways. First, we use copulas to examine asymmetric tail dependence structures, since recent studies demonstrate that the DCC model or the Markov switching model with Gaussian innovations is inappropriate for capturing asymmetric tail dependences. For example, Garcia and Tsafack (2011) point out that the asymmetric DCC model by Cappiello, Engle, and Sheppard (2006) cannot reproduce asymmetric tail dependence in the international equity market, but the Markov switching copula model can. In addition, Okimoto (2008) shows that the Markov switching model with Gaussian innovations that has been used to address asymmetric correlation issues (see Ang and Bekaert, 2002; Ang and Chen, 2002) is statistically rejected in favor of the Markov switching copula model with asymmetric tail dependences. Thus, it is of great importance to model asymmetric tail dependences using asymmetric copulas, as we do here.

Second, instead of considering correlation, we use three copula-based dependence measures, specifically, Spearman's rho, and upper and lower tail dependences to evaluate dependence. Embrechts, McNeil, and Straumann (2002) and Embrechts, Lindskog, and McNeil (2003) emphasize that a correlation is not a good measure of dependence for non-elliptical distributions with asymmetric tail dependence such as those considered in this paper. Third, we allow for a number of dependence regimes ranging from one to four. Most previous studies allow only one simple linear or non-linear trend. It is, however, very questionable whether the dependence trends in international equity markets over the last 35 years can be characterized by one trend, given the many factors affecting the financial market integration mentioned above. Fourth, we investigate the evolutionary paths of upper and lower tail dependences. Given the asymmetries in dependence structures in international equity markets found by a 
number of studies, ${ }^{3}$ this could be a very important extension.

We apply the multiple-regime STCG model to four of the largest equity markets, namely French (FR), German (GE), U.K. (UK), and U.S. (US) markets. The results of our empirical analysis are summarized as follows. First, we confirm the importance of capturing the fattailed nature of stock returns, showing the inappropriateness of the MVN model employed by most previous studies, such as those of Longin and Solnik (1995) and Berben and and Jansen (2005). In addition, our results suggest that the symmetrized Hüsler-Reiss (HR) copula model dominates the Normal and symmetrized Joe-Clayton (JC) copula models. Second, our analysis demonstrates that three dependence regimes are sufficient to describe dependence trends in international equity markets over the last 35 years. Third, our results indicate a significant increase in both upper and lower tail dependences. Fourth, our results provide clear evidence of the asymmetric evolution of upper and lower tail dependences.

Following these empirical findings, we calculate the time series of three copula-based dependence measures to see when the important increases in dependence occurred and which tail contributed most to the increases. The results demonstrate that the FR-GE and FRUK pairs experienced a rapid increase in dependence between 1986 and 1991, and 2000 and 2004, with lower tail dependence playing the more important role in these increases. On the other hand, dependence in the GE-US and FR-US pairs underwent a gradual increase from 1987 onward, whereas the GE-UK and UK-US pairs' dependence increased almost linearly over the entire sample. Furthermore, our results show that for these four pairs upper tail dependence contributed to increasing dependence more than lower tail dependence.

Lastly, we investigate the economic significance of our empirical findings from a risk management point of view based on the $99 \%$ VaR and ES. Our results indicate that both 99\% VaR and ES in 2008 are larger by about 20\% compared to those in 1973. In addition, the benefits from international diversification to decrease risk have almost vanished in recent years. It is, therefore, critical to recognize our finding of increasing trends in dependence with possible asymmetry in international equity markets.

\footnotetext{
${ }^{3}$ See Okimoto (2008) and reference therein for a discussion of the asymmetric dependence in international equity markets.
} 
The remainder of the paper is organized as follows. Section 2 introduces the model and the idea behind our methodology. Section 3 provides the empirical results, followed by some robustness check in Section 4. Implications of our empirical findings for international diversification and risk management are examined in Section 5. Lastly, Section 6 concludes.

\section{Model and Estimation}

The main purpose of this paper is to investigate asymmetric increasing dependence in international equity markets. It is desirable to model the dependence structure and evolutionary process in a flexible way. To this end, we propose using a multiple-regime smooth-transition copula-GARCH (STCG) model. The basic idea behind the model is to use copulas to model the contemporaneous dependence between variables and to specify the dynamics of dependence, or copula parameter(s), with a multiple-regime smooth-transition model.

According to a version of Sklar's (1959) theorem for conditional copulas presented in Patton (2006a), ${ }^{4}$ the conditional joint distribution $H$ of two random variables $X_{1 t}$ and $X_{2 t}$ given some information set $\mathcal{F}_{t-1}$ can be decomposed into two parts: conditional marginal distributions $F_{1}\left(\cdot \mid \mathcal{F}_{t-1}\right)$ and $F_{2}\left(\cdot \mid \mathcal{F}_{t-1}\right)$, describing the conditional marginal behavior of $X_{1 t}$ and $X_{2 t}$, and a conditional copula $C\left(\cdot \mid \mathcal{F}_{t-1}\right)$, representing the dependence structure between $X_{1 t}$ and $X_{2 t}$. More specifically, $H$ can be written as follows:

$$
H\left(x,_{1} x_{2} \mid \mathcal{F}_{t-1} ; \boldsymbol{\theta}\right)=C\left(F_{1}\left(x_{1} \mid \mathcal{F}_{t-1} ; \boldsymbol{\theta}_{1}\right), F_{2}\left(x_{2} \mid \mathcal{F}_{t-1} ; \boldsymbol{\theta}_{2}\right) \mid \mathcal{F}_{t-1} ; \boldsymbol{\theta}_{C}\right)
$$

Here, $\boldsymbol{\theta}_{C}$ is a parameter vector for the copula, $\boldsymbol{\theta}_{1}$ and $\boldsymbol{\theta}_{2}$ are parameter vectors for each marginal distribution, and $\theta=\left(\theta_{1}^{\prime}, \theta_{2}^{\prime}, \theta_{C}^{\prime}\right)^{\prime}$ is a parameter vector for the joint distribution. As a consequence, marginal distributions and a dependence structure can be specified independently with great flexibility. In addition, we model copula parameters using the multiple-regime smooth-transition model. By doing so, we can capture dominant trends in dependence, together with possible regime changes. In the following subsections, we describe models for the marginal distributions, copula, and dynamics of dependence. Then we derive the conditional log-likelihood function to estimate the models via maximum likelihood

\footnotetext{
${ }^{4}$ See Joe (1997) and Nelsen (2006) for details of copula theory.
} 
estimation (MLE).

\subsection{Marginal distributions}

For marginal distributions we use the $\operatorname{GARCH}(1,1)$ model with Normal or Student's $t$ disturbance. Specifically, the model for the marginal distribution can be expressed as

$$
\left\{\begin{array}{l}
x_{i t}=\mu_{i}+\varepsilon_{i t}, \\
\varepsilon_{i t}=\sqrt{h_{i t}} v_{i t}, \\
h_{i t}=\omega_{i}+\alpha_{i} \varepsilon_{i, t-1}^{2}+\beta_{i} h_{i, t-1},
\end{array}\right.
$$

for $t=1, \ldots, T$ and $i=1,2$, where $T$ is the sample size. We assume $v_{i t}$ follows the standard Normal distribution or Student's t-distribution with mean 0, variance 1, and degrees of freedom $\nu_{i}$. This specification is motivated by the well-known fact that the $\operatorname{GARCH}(1,1)$ model (with Student's t-disturbance) is able to capture many features of financial data, such as volatility clustering and fat-tailedness. Since no strong serial correlation is observed in each series, we simply model the conditional expectation as a constant without an AR term. In addition, we do not include the leverage effect term in the GARCH model, since it is not always significant and does not affect the empirical results. ${ }^{5}$ Furthermore, to keep our model tractable, we do not assume a smooth-transition structure in the marginal model. The empirical results, however, are not sensitive to this assumption as we will see in Section 4.

Note that our copula framework allows us to use the $t$-disturbance to capture fattailedness. This is of great importance, as we will confirm in the next section, since the tail behavior of international stock returns may not be well characterized by the Normal GARCH model. With an extra parameter for the tail-thickness, the $t$-GARCH model contains five parameters: $\boldsymbol{\theta}_{i}=\left(\mu_{i}, \omega_{i}, \alpha_{i}, \beta_{i}, \nu_{i}\right)^{\prime}$, while the Normal GARCH model contains four parameters: $\boldsymbol{\theta}_{i}=\left(\mu_{i}, \omega_{i}, \alpha_{i}, \beta_{i}\right)^{\prime}$.

\footnotetext{
${ }^{5}$ We also tried the GARCH model with the leverage effect term. The results are qualitatively similar as those without the leverage effect. To save space, we have not provided the results, but they are available on request from the authors.
} 


\subsection{Copula}

We use the Normal copula as a benchmark. The Normal copula is the copula for the multivariate Normal distribution and is given as follows:

$C^{N O R}\left(u, v ; \delta_{1}\right)=\int_{-\infty}^{\Phi^{-1}(u)} \int_{-\infty}^{\Phi^{-1}(v)} \frac{1}{2 \pi \sqrt{1-\delta_{1}^{2}}} \exp \left\{-\frac{s^{2}-2 \delta_{1} s t+t^{2}}{2\left(1-\delta_{1}^{2}\right)}\right\} d s d t, \delta_{1} \in(-1,1)$.

Here, $\Phi(\cdot)$ is the cumulative distribution function $(\mathrm{CDF})$ of the standard Normal distribution. It is important to note that with the Normal copula and Normal GARCH marginals we obtain the multivariate Normal GARCH model, which has been used in much of the previous literature concerning comovements in stock returns. Note also that in this case $\delta_{1}$ is equivalent to the usual linear correlation between two variables. For this reason, the Normal copula can capture only linear and symmetric dependence. In addition, it can be shown that the Normal copula exhibits no tail dependence. ${ }^{6}$ To investigate the possible asymmetric evolution of tail dependences we employ two other copulas; the symmetrized Joe-Clayton (SJC) copula and the symmetrized Hüsler-Reiss (SHR) copula.

The SJC copula was proposed by Patton (2006a) to model asymmetric exchange rate dependence. It is based on the Joe-Clayton (JC) copula given by

$C^{J C}\left(u, v ; \delta_{1}, \delta_{2}\right)=1-\left(1-\left\{\left[1-(1-u)^{\kappa}\right]^{-\gamma}+\left[1-(1-v)^{\kappa}\right]^{-\gamma}-1\right\}^{-1 / \gamma}\right)^{1 / \kappa}, \delta_{1}, \delta_{2} \in(0,1)$ where $\kappa=1 / \log _{2}\left(2-\delta_{1}\right)$ and $\gamma=1 / \log _{2}\left(\delta_{2}\right)$. With this specification, two copula dependence parameters, $\delta_{1}$ and $\delta_{2}$, for the JC copula are coincident with upper and lower tail dependences, respectively. Patton (2006a) points out that even when the two copula dependence parameters are equal, there is still some (slight) asymmetry in the JC copula and symmetrizes it as

$$
C^{S J C}\left(u, v ; \delta_{1}, \delta_{2}\right)=0.5 \cdot\left\{C^{J C}\left(u, v ; \delta_{1}, \delta_{2}\right)+C^{J C}\left(1-u, 1-v ; \delta_{2}, \delta_{1}\right)+u+v-1\right\}
$$

The SJC copula is a mixture of the JC copula and the survival JC copula. ${ }^{7}$ By construction,

\footnotetext{
${ }^{6}$ For a proof of this statement see Embrechts, McNeil, and Straumann (2003). The definition of upper and lower tail dependences are given in the next section.

${ }^{7}$ The survival copula $C^{\prime}$ of a copula $C$ is defined using a survival function as

$$
C^{\prime}(u, v)=u+v-1+C(1-u, 1-v) .
$$
}


the SJC copula nests symmetry as a special case, making it a more interesting specification for examining the existence of asymmetric dependence, from an empirical perspective, than the JC copula.

The SHR copula is based on the Hüsler-Reiss (HR) copula, whose survival copula was identified as the best copula for describing a bear regime in international equity markets by Okimoto (2008). ${ }^{8}$ The HR copula is expressed as

$$
\begin{aligned}
C^{H R}(u, v ; \delta)=\exp \{\log u & \cdot \Phi\left(\delta^{-1}+\frac{\delta}{2} \log \left(\frac{\log u}{\log v}\right)\right) \\
& \left.+\log v \cdot \Phi\left(\delta^{-1}-\frac{\delta}{2} \log \left(\frac{\log u}{\log v}\right)\right)\right\}, \delta \in(0, \infty)
\end{aligned}
$$

Note that the HR copula has only one parameter, which is monotonically related to the upper tail dependence. ${ }^{9}$ In addition, it is known that the HR copula exhibits no lower tail dependence. In other words, the HR copula is incapable of capturing lower tail dependence and symmetric dependence for any parameter values, which is undesirable from an empirical perspective. Therefore, following Patton (2006a), we symmetrize it as

$$
C^{S H R}\left(u, v ; \delta_{1}, \delta_{2}\right)=0.5 \cdot\left\{C^{H R}\left(u, v ; \delta_{1}\right)+C^{H R}\left(1-u, 1-v ; \delta_{2}\right)+u+v-1\right\} .
$$

As a consequence, the SHR copula has two dependence parameters, $\delta_{1}$ and $\delta_{2}$. While $\delta_{1}$ characterizes upper tail dependence, $\delta_{2}$ captures lower tail dependence.

Although both the SHR and SJC copulas can describe asymmetric dependence, there is one notable difference between the SHR and SJC copulas, which is the maximum degree of tail dependence. By construction, the maximum value for the upper and lower tail dependences for the SHR copula is 0.5 , while that for the SJC copula is 1 . Recall that the Normal copula has no tail dependence. Thus, our model specification can capture a wide variety of tail dependences.

Note that we allow the copula parameters to be time-dependent. The model for the dynamics of dependence is discussed in detail in the next subsection.

\footnotetext{
${ }^{8}$ Okimoto (2008) refers to the survival HR copula as simply the HR copula.

${ }^{9}$ It is not easy to parameterize the HR copula using the upper tail dependence as we do with the JC copula, since the analytical representation for the upper tail dependence coefficient of the HR copula is equal to $2-2 \phi\left(\delta^{-1}\right)$. See Mathieu and Mohammed (2012) for the representation.
} 


\subsection{Dynamics of dependence}

To examine the asymmetric increasing trends in dependence in international equity markets, we need to specify a model for the dynamics of the copula parameters. To this end, we adopt the multiple-regime smooth-transition model. The smooth-transition model is formally analyzed by Teräsvirta (1994) in an autoregressive model framework and is recently applied by Berben and Jansen (2005) and Kumar and Okimoto (2011) to examine correlation dynamics in international equity and bond markets. In what follows, we will use the three-regime smooth-transition model as an example. With this three-regime smooth-transition framework, we can model each copula parameter $\delta_{i}$ ( $i=1$ for the Normal copula and $i=1,2$ for the SJC and SHR copulas) as

$$
\delta_{i t}\left(s_{t} ; \boldsymbol{\theta}_{c i}\right)=\delta_{i}^{(1)}+\left(\delta_{i}^{(2)}-\delta_{i}^{(1)}\right) G^{(1)}\left(s_{t} ; \gamma_{i}^{(1)}, d_{i}^{(1)}\right)+\left(\delta_{i}^{(3)}-\delta_{i}^{(2)}\right) G^{(2)}\left(s_{t} ; \gamma_{i}^{(2)}, d_{i}^{(2)}\right) .
$$

Here, $\boldsymbol{\theta}_{c i}=\left(\delta_{i}^{(1)}, \delta_{i}^{(2)}, \delta_{i}^{(3)}, \gamma_{i}^{(1)}, \gamma_{i}^{(2)}, d_{i}^{(1)}, d_{i}^{(2)}\right)^{\prime}$, and $G(\cdot)$ is a transition function that is modeled by the logistic function as

$$
G\left(s_{t} ; \gamma, d\right)=\frac{1}{1+\exp \left(-\gamma\left(s_{t}-d\right)\right)}, \quad \gamma>0,
$$

where $s_{t}$ is a transition variable, and $\gamma$ and $d$ are smoothness and location parameters, respectively. Note that for the SHR and SJC copula models, we assume that the two copula parameters share the same transition parameters, i.e. $d_{1}^{(j)}=d_{2}^{(j)}, \gamma_{1}^{(j)}=\gamma_{2}^{(j)}$ for $j=1,2$. This assumption is necessary to conduct the hypothesis test for the equality of one of the copula parameters across regimes, without identification problems. Nonetheless, this assumption is innocuous for the purpose of this paper, since the dynamics of two copula parameters can be very different as $\delta_{1}^{(j)}$ are allowed to take different values from $\delta_{2}^{(j)}$ for $j=1,2,3$.

For a transition variable, following Lin and Teräsvirta (1994) and Berben and Jansen (2005), we use a linear time trend; specifically, we set $s_{t}=t / T$. In addition, we assume $0.01 \leq d_{i}^{(1)}<d_{i}^{(2)} \leq 0.99$ so that we can detect the dependence transition within the sample period. Under this assumption, each copula parameter, $\delta_{i t}$, changes smoothly from $\delta_{i}^{(1)}$ via $\delta_{i}^{(2)}$ to $\delta_{i}^{(3)}$ with time, as first the function $G^{(1)}$ changes from 0 to 1 , followed by a similar change in $G^{(2)}$. As a consequence, we can capture dominant trends of dependence 
in international equity markets together with possible regime changes over the last 35 years. Another attractive feature of this framework is that data can choose the best pattern for the transition of copula parameters. The change is abrupt for large values of $\gamma$, while the transition is gradual for small values of $\gamma$. In addition, the location parameter $d$ can adjust the location of the inflection points.

\subsection{Likelihood Function}

Since we have fully specified the model, we can derive the (conditional) log-likelihood function $l(\boldsymbol{\theta})$ to implement the MLE, where $\boldsymbol{\theta}$ is a vector of parameters to estimate. Differentiating the conditional joint distribution (??) with respect to each $x_{i t}$, we can get the conditional joint density $h$ as

$$
\begin{aligned}
h\left(x_{1 t}, x_{2 t} \mid \mathcal{F}_{t-1} ; \boldsymbol{\theta}\right)= & \frac{\partial^{2} H\left(x_{1 t}, x_{2 t} \mid \mathcal{F}_{t-1} ; \boldsymbol{\theta}\right)}{\partial x_{1 t} \partial x_{2 t}} \\
= & \frac{\partial^{2} C\left(F_{1}\left(x_{1 t} \mid \mathcal{F}_{t-1} ; \boldsymbol{\theta}_{1}\right), F_{2}\left(x_{2 t} \mid \mathcal{F}_{t-1} ; \boldsymbol{\theta}_{2}\right) \mid \mathcal{F}_{t-1} ; \boldsymbol{\theta}_{C}\right)}{\partial x_{1 t} \partial x_{2 t}} \\
= & c\left(F_{1}\left(x_{1 t} \mid \mathcal{F}_{t-1} ; \boldsymbol{\theta}_{1}\right), F_{2}\left(x_{2 t} \mid \mathcal{F}_{t-1} ; \boldsymbol{\theta}_{2}\right) \mid \mathcal{F}_{t-1} ; \boldsymbol{\theta}_{C}\right) \\
& \quad \times f_{1}\left(x_{1 t} \mid \mathcal{F}_{t-1} ; \boldsymbol{\theta}_{1}\right) \times f_{2}\left(x_{2 t} \mid \mathcal{F}_{t-1} ; \boldsymbol{\theta}_{2}\right),
\end{aligned}
$$

where $f_{i}\left(\cdot \mid \mathcal{F}_{t-1}\right)$ for $i=1,2$ is the conditional marginal density of $X_{i t}$, and $c$ is the conditional density of a copula defined as

$$
c\left(u, v \mid \mathcal{F}_{t-1} ; \boldsymbol{\theta}_{C}\right)=\frac{\partial^{2} C\left(u, v \mid \mathcal{F}_{t-1} ; \boldsymbol{\theta}_{C}\right)}{\partial u \partial v}
$$

With the use of equations (??) and (??), the log-likelihood function $l(\boldsymbol{\theta})$ can be obtained as

$$
\begin{aligned}
l(\boldsymbol{\theta})= & \sum_{t=1}^{T} \ln c\left(F_{1}\left(x_{1 t} \mid \mathcal{F}_{t-1} ; \boldsymbol{\theta}_{1}\right), F_{2}\left(x_{2 t} \mid \mathcal{F}_{t-1} ; \boldsymbol{\theta}_{2}\right) \mid \mathcal{F}_{t-1} ; s_{t}, \boldsymbol{\theta}_{C}\right) \\
& +\sum_{t=1}^{T} \ln f_{1}\left(x_{1 t} \mid \mathcal{F}_{t-1} ; \boldsymbol{\theta}_{1}\right)+\sum_{t=1}^{T} \ln f_{2}\left(x_{2 t} \mid \mathcal{F}_{t-1} ; \boldsymbol{\theta}_{2}\right)
\end{aligned}
$$

where $\boldsymbol{\theta}_{C}=\boldsymbol{\theta}_{c 1}$ for the Normal copula and $\boldsymbol{\theta}_{C}=\left(\boldsymbol{\theta}_{c 1}^{\prime}, \boldsymbol{\theta}_{c 2}^{\prime}\right)^{\prime}$ for the SJC and SHR copulas. By maximizing it with respect to $\boldsymbol{\theta}=\left(\boldsymbol{\theta}_{1}^{\prime}, \boldsymbol{\theta}_{2}^{\prime}, \boldsymbol{\theta}_{C}^{\prime}\right)^{\prime}$, we can get the MLE of $\boldsymbol{\theta}$ and calculate the Akaike information criterion (AIC) for model comparison. 


\section{Empirical Results}

This study is based on weekly total market price index data, denominated in US dollars, for four of the largest equity markets, namely the French, German, U.S., and U.K markets. The data are obtained from Datastream with a sample period running from January, 1973 to June, 2008. To achieve our goal of examining asymmetric increasing trends in dependence in international equity markets, the markets investigated should be representative of total markets and should be reasonably well integrated throughout the sample period; this is arguably the case for the four markets investigated.

To make a comprehensive comparison, we consider the following four STCG models: Multivariate Normal model (MVN model), Normal copula model with Student- $t$ margins (NC model), SHR copula model with Student- $t$ margins (SHRC model), and SJC copula model with Student- $t$ margins (SJCC model). Note that the superior performance of the $t$ GARCH model over the Normal GARCH model is evident, and we use the Normal GARCH specification only for the MVN model. We set the number of regimes from one to four for each model, generating sixteen models in total. By estimating these models, we can compare a wide variety of dependence evolutions and empirically choose the best dynamics for the international stock return data. We can then attempt to answer the questions addressed in the Introduction.

\subsection{Model comparison}

In this section, we compare the estimation results from four STCG models. We fit these models to the pairs of stock returns taken from FR, GE, UK, and US equity markets. In other words, all models are estimated for six different country-pairs. In addition, we allow each model to have multiple regimes ranging from one to four. Thus, we estimate sixteen models for six pairs.

Table 1 reports the best fitting model based on the AIC, and the AIC value for each STCG model and each pair. The best model among sixteen models is indicated by bold face. There are a number of observations that should be emphasized. First, AICs of the 
MVN model with Normal GARCH margins are much larger than those of other models with t-GARCH margins for all pairs. In addition, although each estimation result is not reported here to save space, the degrees of freedom for the $t$-distribution is estimated as less than 10 for most of the cases. These results suggest the importance of capturing the fat-tailedness of marginal distributions.

Second, the results indicate that two and three regime models are chosen as the best model for three pairs each. In other words, neither one- nor four- regime models are the best models for all country-pairs, meaning that two or three regimes can describe the dependence trends in international equity market over the last thirty-five years.

Lastly, the table also provides a strong indication of the dominance of the SHRC model over the other three models. The SHRC model is chosen as the best model for five out of six pairs. This clearly suggests the importance of capturing asymmetric trends in tail dependences, which we will formally test in the next subsection. Although the SJCC model can also describe asymmetric tail-dependence evolution, it seems to have too much tail dependence, worsening the fit compared to the SHRC model for all cases. ${ }^{10}$

In sum, the conclusions of our comprehensive model comparison are clear: Capturing the fat-tailedness of marginal distributions is indispensable for modeling international stock returns. In addition, the three dependence regimes sufficiently describe dependence trends in international equity markets over the last 35 years. Furthermore, the SHRC model dominatly outperforms the other models. Given these observations, in the following subsections, we will use the best fitting SHRC model- specifically the three-regime model for the FR-GE and FR-UK pairs and the two-regime model for the other pairs- to investigate the time evolution of dependence in international equity markets in detail, as we seek answers to the four remaining questions posed in the Introduction.

\footnotetext{
${ }^{10}$ Recall that, by construction, the maximum value of upper and lower tail dependences is 0.5 for the SHR copula and 1 for the SJC copula.
} 


\subsection{Hypothesis tests}

In this subsection, we conduct two hypothesis tests to answer questions (iii) and (iv). The first hypothesis test examines whether there has been a significant increase in dependence in international equity markets over the last 35 years. To this end, we conduct a Wald test of the equality of the upper and lower tail copula parameters between the first and last regimes. Thus, the null hypothesis can be expressed as $H_{0}: \delta_{i}^{(1)}=\delta_{i}^{(2)}$ for the two regime model or $H_{0}: \delta_{i}^{(1)}=\delta_{i}^{(3)}$ for the three regime model. Correspondingly, the alternative hypothesis can be written as $H_{1}: \delta_{i}^{(1)} \neq \delta_{i}^{(2)}$ for the two regime model or $H_{1}: \delta_{i}^{(1)} \neq \delta_{i}^{(3)}$ for the three regime model.

The second test concerns the asymmetric trends in dependence. We conduct a Wald test of the equality of the upper and lower tail copula parameters across each regime. In other words, the null hypothesis of the test is $H_{0}: \delta_{1}^{(j)}=\delta_{2}^{(j)}$, while the corresponding alternative hypothesis is $H_{1}: \delta_{1}^{(j)} \neq \delta_{2}^{(j)}$.

Table 2 reports the estimates for the SHR copula parameters for each regime and the $p$ values of the two tests as stated above. As can be seen from the table, the estimation results indicate that the latter regime has larger copula parameters for all pairs. For instance, for the FR-GE pair, the upper tail copula parameter is estimated as 1.05 for the first regime, 2.37 for the second regime, and 5.30 for the third regime, while the lower tail copula parameter for each regime is estimated as $0.92,1.47$, and 6.56 , respectively. Thus, the estimation results suggest that international equity markets have become more interdependent in recent years. This statement is formally tested by the first test. The $p$-values of the test can be found in the last column of Table 2. As can be seen, all $p$-values are less than 0.05, meaning there are significant increasing trends in dependence in both tails for all country pairs.

This result seems to be in contrast to the recent finding by Bekaert, Hodrick, and Zhang (2009), of no significant upward trend in cross-country correlations. However, their estimates of the trends in correlation for G7 countries indicate that correlations in G7 countries have increased by at least 0.18 during the last 26 years, which is consistent with our finding of increasing trends in dependence. We suspect that the difference most likely comes from the 
low power of their tests due to a nonparametric framework and a relatively small sample with 52 observations.

Regarding the asymmetric evolution, the evidence is less clear, but the results still suggest the importance of accommodating asymmetric dependence. As can be seen from the $p$ values of the second test shown in the last row of each pair's result in Table 2, five out of six country pairs exhibit some asymmetry in at least one regime at the $5 \%$ significance level. In particular, four pairs indicate asymmetric dependence in either the first or middle regime. Note also that these asymmetries have become negligible in the latter regime for three country pairs. As a consequence asymmetric dependence is insignificant in the most recent regime for four out of six pairs. We, therefore, conclude that allowing asymmetric dependence is important, particularly in earlier periods, to describe the dependence trends in international equity markets.

\subsection{Dynamics of dependence}

In the last subsection, we showed that there have been significant increases in dependence in international equity markets and dependence evolution could be asymmetric between the upper and lower tails of the joint distribution. In this subsection, we investigate the dynamics of dependence in international equity markets over the last 35 years to see when the important increases occurred and which tail contributed most to the increases. To this end, we calculate three copula-based dependence measures, Spearman's rho, and upper and lower tail dependences, at each time based on the estimated time series of copula parameters obtained from the best fit SHRC model. ${ }^{11}$ By doing so, we can evaluate three aspects of dependence and in a more appropriate way than using only one non-copula-based measure, namely linear correlation. As emphasized by Embrechts, McNeil, and Straumann (2002) and Embrechts, Lindskog, and McNeil (2003), a linear correlation is not a good measure of dependence for a non-elliptical distribution model such as our SHRC model.

Spearman's rho is sometimes called rank correlation, since it can be interpreted as the

\footnotetext{
${ }^{11}$ Note that the estimated time series of copula parameters can be obtained using equation (??), for example.
} 
linear correlation between some "ranks" of the data. Unlike linear correlation, Spearman's rho satisfies all conditions for a measure of concordance proposed by Scarsini (1984). It is, therefore, a reasonable alternative to linear correlation as a measure of global dependence for non-elliptical distributions.

Spearman's rho is defined to be proportional to the probability of concordance minus the probability of discordance for the two vectors $\left(X_{1}, Y_{1}\right)$ and $\left(X_{2}, Y_{3}\right)$, i.e., a pair of vectors with the same marginals, but one vector has a joint distribution with copula $C$, while the components of the other are independent. It can also be considered to be the linear correlation between $F_{X}(X)$ and $F_{Y}(Y)$, which can be calculated from a copula as follows:

$$
\begin{aligned}
\rho_{S} & =3\left\{\operatorname{Prob}\left[\left(X_{1}-X_{2}\right)\left(Y_{1}-Y_{3}\right)>0\right]-\operatorname{Prob}\left[\left(X_{1}-X_{2}\right)\left(Y_{1}-Y_{3}\right)<0\right]\right\} \\
& =\frac{\operatorname{Cov}\left(F_{X}(X), F_{Y}(Y)\right)}{\sqrt{\operatorname{Var}\left(F_{X}(X) \cdot \operatorname{Var}\left(F_{Y}(Y)\right)\right.}} \\
& =12 \int_{0}^{1} \int_{0}^{1} C(u, v) d u d v-3 .
\end{aligned}
$$

Tail dependence measures the dependence in the upper-right-quadrant or lower-leftquadrant tail of a bivariate distribution. It is a concept that is relevant to dependence in extreme values. The definition of upper (lower) tail dependence is the probability that one variable takes an extremely large positive (negative) value, given that the other variable took an extremely large positive (negative) value. The upper tail dependence can be equivalently defined in terms of copulas as follows:

$$
\begin{aligned}
\lambda_{U} & =\lim _{u \uparrow 1} \operatorname{Prob}\left[X \geq F_{X}^{-1}(u) \mid Y \geq F_{Y}^{-1}(u)\right] \\
& =\lim _{u \uparrow 1} \operatorname{Prob}\left[Y \geq F_{Y}^{-1}(u) \mid X \geq F_{X}^{-1}(u)\right] \\
& =\lim _{u \uparrow 1} \frac{1-2 u+C(u, u)}{1-u}
\end{aligned}
$$

provided the limit exists. Similarly, the lower tail dependence can be defined as

$$
\begin{aligned}
\lambda_{L} & =\lim _{u \downarrow 0} \operatorname{Prob}\left[X \leq F_{X}^{-1}(u) \mid Y \leq F_{Y}^{-1}(u)\right] \\
& =\lim _{u \downarrow 0} \operatorname{Prob}\left[Y \leq F_{Y}^{-1}(u) \mid X \leq F_{X}^{-1}(u)\right] \\
& =\lim _{u \downarrow 0} \frac{C(u, u)}{u} .
\end{aligned}
$$


A bivariate copula $C$ has upper (lower) tail dependence if $\lambda_{U} \in(0,1]\left(\lambda_{L} \in(0,1]\right)$ and no upper (lower) tail dependence if $\lambda_{U}=0\left(\lambda_{L}=0\right)$.

Using these three copula-based measures, we can examine three aspects of dependence; general dependence among stock returns measured by Spearman's rho, and dependence between the joint extreme positive (negative) returns evaluated by the upper (lower) tail dependence. In addition, our smooth-transition copula framework allows us to evaluate these three measures in each time period. As a consequence, we can investigate the dynamics of dependence and understand the sources of dependence changes in detail.

Figure 1 plots the time series of three dependence measures implied by the best fitting SHRC model. As can be seen from the figure, three groups share a similar pattern of dependence evolution. The first group consists of the FR-GE and FR-UK pairs, whose best fit model is the three-regime SHRC model. This group experienced a rapid increase in dependence between 1986 and 1991, and 2000 and 2004. The second increase in dependence is consistent with results of Cappiello et al. (2006) and Garcia and Tsafack (2011) who find an increase in dependence after the introduction of the Euro currency. It is clear that lower tail dependence played an important role in the second increase for both pairs. However, there is some difference in the first increase between the two pairs. For the FR-GE pair, upper tail dependence was slightly more influential than lower tail dependence, whereas for the FR-UK pair, lower tail dependence was the most influential.

The second group includes the FR-US and GE-US pairs. These pairs underwent a gradual increase in dependence from 1987, with a larger increase in upper tail dependence than in lower tail dependence. For the FR-US pair, upper tail dependence at the beginning of the sample period was 0.06, which was less than half of the lower tail dependence. However, upper tail dependence rose to 0.33 by the end of the sample period, making it slightly higher than the end-of-sample lower tail dependence of 0.31 . The GE-US pair shows a more remarkable increase in upper tail dependence; rising from 0 at the beginning, to 0.35 at the end of the sample period, and exceeding lower tail dependence by 0.10 .

The remaining two pairs constitute the last group. The GE-UK and UK-US pairs' dependence increased almost linearly over the entire sample period. Like the second group, upper 
tail dependence played a more active role for this group. In particular, for the GE-UK pair upper tail dependence always exceeded lower tail dependence and the difference extended from 0.02 to 0.1 during the sample period. For the UK-US pair, upper and lower tail dependences were 0.05 and 0.13 , respectively, at the beginning of the sample period, while both were 0.30 at the end of the sample.

In sum, our analysis shows that there are three types of asymmetric increasing trends in dependence in international equity markets, confirming the usefulness of our framework for describing a wide variety of dependence dynamics in international equity markets. For the FR-GE and FR-UK pairs, lower tail dependence contributed more to the significant dependence increase, while for the remaining pairs, upper tail dependence was the main cause of increasing dependence. This indicates that it is important to consider not only the lowertail dependence, or contagion, but also upper-tail dependence to analyze the dependence evolution in equity markets. Investigating the sources of the increases in tail dependences would be interesting, but is beyond the scope of the paper and left for future research.

\section{Robustness Checks}

In the previous section, we demonstrated asymmetric increasing trends in dependence in international equity markets. In this section, we check the robustness of our finding with respect to the currency denomination and marginal model specification.

\subsection{Currency denomination}

Our empirical results are based on weekly total market price index data, denominated in US dollars, for FR, GE, UK, and US. This currency denomination is relevant because US investors considering international asset allocation or risk management problems are focused on stock returns in US dollars. However, a natural question arising from the use of US dollardenominated returns is that our finding of increasing dependence in international equity markets could be an artefact of currency unification. For instance, when the US dollar appreciates, stock returns in US dollars for countries other than the US tend to be small, generating positive correlations between stock returns in US dollars. If this tendency becomes 
stronger as exchange rate markets develop, the result would be increasing dependence, as we mentioned in previous sections. It is, therefore, very instructive to check the robustness of our results against the currency used in the analysis.

To examine the effects of currency on our results, we fit the same SHRC models as those used in the subsections 3.2 and 3.3 to the stock returns in local currency. Table 3 reports the estimates for the SHR copula parameters for each regime and the $p$-values for the tests discussed in subsection 3.2. As can be seen, the estimation results indicate the same story as before. The latter regime always has larger copula parameters for all pairs and these increases are statistically significant at the $5 \%$ significance level. In addition, all country pairs, except the UK-US pair, demonstrate asymmetric dependence in either the first or middle regime at the 10\% significance level. However, only GE-UK and GE-US pairs show significant asymmetric dependence in the most recent regime.

We also calculate three copula-based dependence measures for each time period, based on the estimation results from the SHRC model using international stock returns in local currency, and plot them in Figure 2. Again these graphs are fairly consistent with those in Figure 1. The FR-GE and FR-UK pairs have experienced rapid increases in dependence between 1986 and 1991, and 2000 and 2004, although the upper tail dependence shows larger increases than the lower tail dependence for the FR-GE pair. Other country pairs have undergone either gradual increases from 1987 or almost linear increases in dependence, with upper tail dependence playing a key role except for the UK-US pair, for which the upper and lower tail dependences have increased almost equally.

In sum, the results based on international stock returns in local currencies indicate that our findings of asymmetric increasing trends in the lower and upper tail dependences are not driven by currency unification.

\subsection{Marginal model specifications}

The analysis so far assumed that the marginal model is not time-varying. We impose this assumption for several reasons. First, modeling the time-varying dependence is of primary importance for the main purpose of the paper, which is to examine asymmetric increasing 
trends in dependence in international equity markets. Second, including time variation with the marginal model makes it formidable to estimate the model via MLE with many extra parameters to estimate. Third, the financial market integration discussed in the Introduction should be more influential in the joint behaviors of international stock returns than the marginal behaviors. In other words, we have a stronger reason to adopt a time-varying dependence model rather than a time-varying marginal model. For these reasons, we believe that our assumption of a time-invariant marginal model is acceptable as a first investigation. However, it is important to recognize that if we use a misspecified model for the marginal distributions, then any copula model will automatically be misspecified as emphasized by Patton (2006a). It is, therefore, critical to examine whether our finding of asymmetric increasing trends in dependence in international equity markets is robust with respect to the assumption of time-invariant marginal model.

To examine the effects of marginal model specifications on our results, we consider the same SHRC models used in the subsections 3.2 and 3.3 but with a two-state smoothtransition marginal model. Specifically, we assume that all parameters in the marginal model (??) are time-varying, following a two-state smooth-transition model with a common transition function. One problem associated with this model is a difficulty in estimating the model via MLE due to a large number of parameters to estimate. To overcome this difficulty, we employ a multi-stage maximum likelihood estimator (MSMLE), as analyzed by Joe (2005) and Patton (2006b). The MSMLE is obtained by getting the MLE for the parameters for marginal and copula models separately. More precisely, to get the MSMLE, we first calculate the MLE for each marginal model, then calculate the MLE for copula parameters using the quasi-likelihood with estimated marginal parameter in the first stage. Joe (2005) shows that the MSMLE generally has good asymptotic relative efficiency compared with the MLE based on numerical comparisons.

Figure 3 plots the time series of three dependence measures implied by the SHRC models with the two-state smooth-transition marginal model. As can be seen, the basic features are the same as those in Figure 1, although there are some differences in the contribution of the upper and lower tail dependences: The FR-GE and FR-UK pairs show rapid increases in 
dependence between 1986 and 1991, and 2000 and 2004, while other country pairs demonstrate either gradual increases from 1987 or almost linear increases in dependence. Thus, the results suggest that our findings of asymmetric increasing trends in dependence are not an artefact of a misspecified marginal model, but are a genuine feature in international equity markets.

\section{Implications for the risk management}

In the previous sections, we documented clear evidence of increasing trends in dependence with asymmetry between upper and lower tail dependences in international equity markets. In this section, we evaluate the economic significance of our empirical results for the risk management.

Following Guidolin and Timmermann (2006) and Okimoto (2008), we assess the economic significance based on VaR and ES ratios. This is relevant because comovements in international stock returns play a crucial role in the evaluation of risk measures, such as $99 \%$ VaR, $\operatorname{VaR}(0.99)$, which is defined as the 99 percentile point of a portfolio's loss distribution, and $99 \% \mathrm{ES}, \mathrm{ES}(0.99)$, which is defined as the expected loss conditional on the loss exceeding the $\operatorname{VaR}(0.99)$. Since VaR and ES heavily depend on the lower tail dependence between stock returns, the asymmetric increasing trends in dependence should greatly influence the calculation of these measures. ${ }^{12}$

We calculate the portfolio minimizing the one week $\operatorname{VaR}(0.99)$ at the end of June every 5 years, based on the estimation results from the best fitting SHRC model for each pair. ${ }^{13}$ Then we evaluate the ratio of $\operatorname{VaR}(0.99)$ for every 5 year period to $\operatorname{VaR}(0.99)$ in 1973 , the first year in the sample, to investigate the evolution of risk in international equity markets over the last 35 years. In addition, we calculate the $\operatorname{VaR}(0.99)$ ratio for the portfolio minimizing $\operatorname{VaR}(0.99)$

\footnotetext{
${ }^{12}$ We also conducted the same analysis using the upper tail of the stock return distribution as our results indicate that the tails are not symmetrical. We found that increases in the risk measures are qualitatively similar but a little stronger. Our results are omitted to save space but are available on request from the authors.

${ }^{13}$ For this calculation, we only consider portfolios with nonnegative weights in each stock. In other words, we do not allow short positions.
} 
to a portfolio that invests entirely in the least risky country with smaller $\operatorname{VaR}(0.99) .{ }^{14} \mathrm{By}$ doing so, we can examine how the benefits from international diversification changed over time. Furthermore, we conduct the same analysis using the $\mathrm{ES}(0.99)$ ratio as another measure of risk.

To calculate the portfolio minimizing $\operatorname{VaR}(0.99)$ and $\operatorname{ES}(0.99)$, we generate 100,000 data using the estimated best SHRC model for international stock returns in US dollars and numerically solve the minimization problem. For this purpose, we have to generate a random vector $(U, V)^{\prime}$ from the HR and survival HR copulas, since the SHR copula is a mixture of these two copulas. This can be done using a conditional copula, which can be calculated as a partial derivative of $C$ with respect to the first variable:

$$
C_{2 \mid 1}(v \mid u)=\frac{C(u, v)}{\partial u} .
$$

For the HR copula, this can be expressed as

$$
C_{2 \mid 1}^{H R}(u \mid v ; \delta)=C^{H R}(u, v ; \delta) \cdot u^{-1} \Phi\left(\delta^{-1}+0.5 \delta \log \left(\frac{\log u}{\log v}\right)\right),
$$

from which the conditional copula of the survival HR copula can be also easily calculated. By definition, if $U \sim U(0,1)$ and $V \sim C_{2 \mid 1}(\cdot \mid U)$, then $(U, V) \sim C$. Hence, if $U$ and $Q$ are independent $U(0,1)$ random variables and define $V$ so that $C_{2 \mid 1}(V \mid U)=Q$, then $(U, V) \sim$ $C$. Once we obtain $(U, V)^{\prime}$ from the SHR copula, the desired data can be produced by transforming $U$ and $V$ with the inverse of the CDF of each marginal distribution. Note that for a marginal distribution, we use the estimated unconditional distribution to capture only the effect of dependence evolution.

Figure 4 plots the VaR and ES ratios of $\operatorname{VaR}(0.99)$ and $\operatorname{ES}(0.99)$ every 5 years relative to the 1973 values. As can be seen, both ratios show a general increase over the last 35 years for all country pairs, meaning that the $99 \%$ VaR and ES have increased in more recent years. In particular, the VaR ratio in 2008 ranges from 1.11 to 1.29 with a mean of 1.20 , while the ES ratio in 2008 is between 1.08 and 1.28, and is 1.19 on average. Thus the $99 \%$ VaR (ES) in 2008 exceeds that in 1973 by $11 \%$ (8\%) to $29 \%$ (28\%) with a mean of $20 \%(19 \%)$.

\footnotetext{
${ }^{14}$ The least risky countries are France, UK, US, UK, US, and US for the FR-GE, FR-UK, FR-US, GE-UK, GE-US, and UK-US pairs, respectively.
} 
The VaR and ES ratios for the minimized $\operatorname{VaR}(0.99)$ and $\operatorname{ES}(0.99)$ relative to those of the least risky country are depicted in Figure 5. Similar to Figure 4, both ratios mostly increase to 1 for all country pairs, indicating that the benefits from international diversification for the risk management have diminished over the sample period. For instance, by allocating our assets optimally to France and Germany in the sense of minimizing $99 \%$ VaR (ES), we can reduce the $\operatorname{VaR}(0.99)$ by $21 \%$ (19\%) in 1973 compared with investing all our assets in France, but only $2 \%(3 \%)$ in 2008 .

Given the fact that the $99 \% \mathrm{VaR}$ and ES are the most widely used risk measures, these changes are important. If a fund manager overlooked the changes in these risk measures, he/she would have substantially underestimated risk, causing a serious problem for a fund with nontrivial probability. It is, therefore, essential to recognize the paper's finding of increasing trends in dependence with possible asymmetry for the risk management in international equity markets.

\section{Conclusion}

In this paper, we investigated the asymmetric increasing trends in dependence in international equity markets over the last 35 years. In particular, the paper addressed several important empirical questions, including the number of dependence regimes and the existence of asymmetry in dependence trends. To find answers to these questions, we developed a multiple-regime smooth-transition copula-GARCH (STCG) model. More specifically, we estimated a multivariate Normal model (MVN model), a Normal copula model with Student$t$ margins (NC model), a SHR copula model with Student- $t$ margins (SHRC model), and a SJC copula model with Student-t margins (SJCC model). Then we compared them to find the suitable evolution of dependence in international equity markets. Through the comprehensive comparison of these competing specifications, we selected the SHRC model as the best model for describing the dependence evolution in international equity markets over the last 35 years. The results offer several important implications. First, the fat-tailedness of marginal distributions is indispensable, indicating the inappropriateness of the MVN model. 
Second, two or three dependence regimes are sufficient for describing the dynamics of dependence in international equity markets. Third, capturing the asymmetric evolution between upper and lower tails is substantial, suggesting the importance of using non-elliptical copula, such as the SHR copula.

Based on the best fitting SHRC model, the paper conducted two hypothesis tests to formally examine increases, and the existence of asymmetry, in dependence trends. The results indicated that both upper and lower tail dependences increased significantly during the last 35 years. In addition, our statistical tests confirmed that during the early period of the sample there was some asymmetry in the comovement between lower and upper tails for most country pairs, but this asymmetry has typically disappeared by the end of the sample period.

Next, we provided the implied time series of three copula-based dependence measures from the best fitting SHRC model to identify when the important dependence increases occurred and which tail dependence contributed most to these increases. The results showed that the FR-GE and FR-UK pairs experienced rapid increases in dependence between 1986 and 1991, and 2000 and 2004, and that lower tail dependence played the most important role in these increases. On the other hand, the GE-US and FR-US pairs saw a gradual increase in dependence from 1987, whereas the GE-UK and UK-US pairs' dependence increased almost linearly over the entire sample period. Furthermore, the results suggested that for these pairs upper tail dependence contributed more than lower tail dependence to increasing dependence. We also demonstrated that our finding of asymmetric increasing trends in dependence is mostly robust with respect to the currency denomination and specification of the marginal model.

As a final contribution to studies in the field of dependence in international equity markets, this paper investigated the economic significance of our empirical findings from a risk management point of view, based on the 99\% Value at Risk and expected shortfall. Our results indicate that both risk measures have increased by about $20 \%$ over the last 35 years due to the diminishing benefits from international diversification to decrease risk. In particular, the benefits from international diversification had almost vanished by 2008. Thus, the 
paper's finding of increasing trends in dependence with possible asymmetry in international equity markets has significant implications for the risk management.

These conclusions also raise several topics for future investigation. One such topic is the scope to pursue the economic factors behind dependence evolution in international equity markets. Our results show there was a marked increase in dependence around 1987 for most of the country pairs studied, suggesting that the 1987 stock market crash played some role in the increase as suggested by Von Furstenberg and Jeon (1989) and Koch and Koch (1991). If this role is confirmed, the recent simultaneous global drop in stock markets could result in another increase in dependence between international equity markets. Examining this possibility by clarifying the economic factors contributing to dependence evolution is a challenge for future work. A recent study by Engle and Rangel (2008) investigates the global macroeconomic causes of volatility fluctuation using the implied volatility time series from the Spline GARCH model. Similar methodology could be used to identify the economic factors of dependence evolution.

Another topic for future studies would relate to the development of a multi-dimensional framework to investigate dependence evolution in international markets. This paper employed the pairwise analysis as used by Longin and Solnik (2001) and Okimoto (2008), which is satisfactory for initial investigation. However, to analyze the dynamics of dependence more precisely, a multi-dimensional model would be more desirable. There are at least two problems associated with the multivariate extension. The first problem is that the number of parameters increases quickly as the number of variables increases. For instance, the four-variate three-regime NC model has 42 parameters to be estimated. In other words, we have to maximize the log-likelihood function with respect to 42 parameters, which is not an easy task. One possibility to mitigate this problem is to develop a MCMC algorithm for the multiple-regime STCG model. Another problem is that few flexible multivariate non-elliptical copulas are available. As we confirmed, accommodating asymmetric dependence could be important for analyzing dependence evolution in international equity markets. Recently, some flexible multivariate copula models including vine copulas and hierarchical Archimedean copulas are developped. Applying these copulas and examining asymmetric in- 
creasing trends in dependence across several countries including the emerging markets would be a fruitful endeavor.

\section{Acknowledgments}

We thank an editor and two anonymous referees for there thoughtful comments and suggestions. We are also indebted to James Hamilton and seminar participants at the 2010 JSM meeting, the Bank of Japan, University of Tokyo, Hitotsubashi University, Kyoto University, Osaka University, Statistics and Econometrics Symposium in Kyoto, and the Tokyo Finance Workshop for their valuable comments and suggestions.

\section{References}

[1] Ang, A. Bekaert, G., 2002. International asset allocation with regime shifts, Review of Financial Studies 15, 1137-1187.

[2] Ang, A., Chen, J., 2002. Asymmetric correlations of equity portfolios, Journal of Financial Economics 63, 443-494.

[3] Bekaert, G., Hodrick, R.J., Zhang, X., 2009. International stock return comovements. Journal of Finance 64(6), 2591-2626.

[4] Berben, R.-P., Jansen, W.J., 2005. Comovement in international equity Markets: A sectoral view, Journal of International Money and Finance 24, 832-857.

[5] Cappiello, L., Engle, R. F., Sheppard, K., 2006. Asymmetric dynamics in the correlations of global equity and bond returns, Journal of Financial Econometrics 4, 537-572.

[6] Christoffersen, P., Errunza, V., Jacobs, K., Langlois, H., 2012. Is the potential for international diversification disappearing? A dynamic copula approach, Review of Financial Studies 25(12), 3711-3751. 
[7] Embrechts, P., Lindskog, F., McNeil, A., 2003. Modelling dependence with copulas and applications to risk management, in: Rachev, S. (Eds.), Handbook of Heavy Tailed Distributions in Finance. Elsevier, North Holland, pp. 329-384.

[8] Embrechts, P., McNeil, A., Straumann, D., 2002. Correlation and dependence properties in risk management: Properties and pitfalls, in: Dempster, M. (Eds.), Risk Management: Value at Risk and Beyond. Cambridge University Press, Cambridge, pp. 176-223.

[9] Engle, R.F., Rangel, J.G., 2008. The spline-GARCH model for low-frequency volatility and its global macroeconomic causes. Review of Financial Studies 21(3), 1187-1222.

[10] Garcia, R., Tsafack, G., 2011. Dependence structure and extreme comovements in international equity and bond markets, Journal of Banking and Finance 35, 1954-1970.

[11] Guidolin, M., Timmermann, A., 2006. Term structure of risk under alternative econometric specifications. Journal of Econometrics 131, 285-308.

[12] Joe, H., 1997. Multivariate Models and Dependence Concepts. Chapman \& Hall. New York.

[13] Joe, H., 2005. Asymptotic efficiency of the two-stage estimation method for copula-based models, Journal of Multivariate Analysis 94, 401-419.

[14] King, M., Sentana, E., Wadhwani, S., 1994. Volatility and links between national stock markets. Econometrica 62, 901-933.

[15] Koch, P.D., Koch, T.W., 1991. Evolution in dynamic linkages across national stock indexes. Journal of International Money and Finance 10, 231-251.

[16] Kose, M. A., Prasad, E., Rogoff, K., Wei, S.-J., 2009. Financial globalization: A reappraisal. IMF Staff Papers 56, 8-62.

[17] Kumar, M.S., Okimoto, T., 2011. Dynamics of international integration of government securities' markets. Journal of Banking and Finance 35, 142-154. 
[18] Lane, P.R., Milesi-Ferretti, G.M., 2007. The external wealth of nations mark II: Revised and extended estimates of foreign assets and liabilities, 1970-2004. Journal of International Economics 73, 223-250.

[19] Lin, C.-F.J., Teräsvirta, T., 1994. Testing the constancy of regression parameters against continuous structural change. Journal of Econometrics 62, 211-228.

[20] Longin, F., Solnik, B., 1995. Is the correlation in international equity returns constant: 1960-1990? Journal of International Money and Finance 14, 3-26.

[21] Longin, F., Solnik, B., 2001. Extreme correlation of international equity markets, Journal of Finance 56, 649-676.

[22] Mathieu, R., Mohammed, S., 2012. Extreme value copulas and max-stable processes. Journal de la Société Française de Statistique 154, 138-150.

[23] Nelsen, R.B., 2006. An Introduction to Copulas, second ed. Lecture Notes in Statistics, Springer, Verlag, New York.

[24] Okimoto, T., 2008. New evidence of asymmetric dependence structures in international equity markets. Journal of Financial and Quantitative Analysis 43(3), 787-816.

[25] Patton, A., 2006a. Modelling asymmetric exchange rate dependence. International Economic Review 47(2), 527-556.

[26] Patton, A., 2006b. Estimation of multivariate models for time series of possibly different lengths. Journal of Applied Econometrics 21(2), 147-173.

[27] Scarsini, M., 1984. On measures of concordance. Stochastica 8, 201-218.

[28] Sklar, A., 1959. Fonctions de répartition à $n$ dimensions et leurs marges. Publications de l'Institut de Statistique de l'Université de Paris 8, 229-231.

[29] Teräsvirta, T., 1994. Specification, estimation, and evaluation of smooth transition autoregressive models. Journal of the American Statistical Association 89, 208-218. 
[30] Von Furstenberg, G.M., Jeon, B.N., 1989. International stock prices movements: Links and messages. Brookings Papers on Economic Activity 1, 125-179. 
Table 1: Result of model comparison

\begin{tabular}{|c|c|c|c|c|c|c|c|}
\hline Model & & FR-GE & FR-UK & FR-US & GE-UK & GE-US & UK-US \\
\hline \multirow{2}{*}{ MVN } & AIC & 15941.36 & 16631.721 & 16379.42 & 16288.18 & 15906.05 & 15992.72 \\
\cline { 2 - 8 } & Best & 3 regimes & 3 regimes & 3 regimes & 2 regimes & 3 regimes & 2 regimes \\
\hline \multirow{2}{*}{$\begin{array}{c}\text { Normal } \\
\text { copula }\end{array}$} & AIC & 15853.97 & 16515.695 & $\mathbf{1 6 2 8 5 . 6}$ & 16182.55 & 15803.85 & 15917.18 \\
\cline { 2 - 8 } & Best & 4 regimes & 4 regimes & $\mathbf{3}$ regimes & 2 regimes & 3 regimes & 2 regimes \\
\hline \multirow{2}{*}{$\begin{array}{c}\text { SHR } \\
\text { copula }\end{array}$} & AIC & $\mathbf{1 5 8 4 8 . 0 3}$ & $\mathbf{1 6 5 0 9 . 5 4 4}$ & 16287.72 & $\mathbf{1 6 1 5 7 . 0 9}$ & $\mathbf{1 5 7 9 8 . 4 6}$ & $\mathbf{1 5 9 0 9 . 7}$ \\
\cline { 2 - 8 } & Best & $\mathbf{3}$ regimes & $\mathbf{3}$ regimes & 2 regimes & $\mathbf{2}$ regimes & $\mathbf{2}$ regimes & $\mathbf{2}$ regimes \\
\hline \multirow{2}{*}{$\begin{array}{c}\text { SJC } \\
\text { copula }\end{array}$} & AIC & 15888.46 & 16544.701 & 16296.77 & 16187.24 & 15825.36 & 15922.85 \\
\cline { 2 - 8 } & Best & 4 regimes & 3 regimes & 3 regimes & 3 regimes & 2 regimes & 2 regimes \\
\hline
\end{tabular}

Note: The table reports the best fitting model based on the AIC and its AIC value for each STCG model and each country pair. The best fitted model among entire models is indicated by bold face. 
Table 2: Results of hypothesis testing (US dollars)

\begin{tabular}{|c|c|c|c|c|c|}
\hline & & $\delta_{i}^{(1)}$ & $\delta_{i}^{(2)}$ & $\delta_{i}^{(3)}$ & $\begin{array}{c}p \text {-value for } \delta_{i}^{(1)}=\delta_{i}^{(2)} \text { or } \\
\delta_{i}^{(1)}=\delta_{i}^{(3)}\end{array}$ \\
\hline \multirow{3}{*}{ FR-GE } & upper-tail parameter & 1.052 & 2.367 & 5.299 & 0.000 \\
\hline & lower-tail parameter & 0.920 & 1.467 & 6.559 & 0.000 \\
\hline & $p$-value for $\delta_{1}^{(j)}=\delta_{2}^{(j)}$ & 0.662 & 0.010 & 0.276 & \\
\hline \multirow{3}{*}{ FR-UK } & upper-tail parameter & 1.320 & 2.021 & 4.427 & 0.000 \\
\hline & lower-tail parameter & 0.635 & 1.644 & 3.788 & 0.000 \\
\hline & $p$-value for $\delta_{1}^{(j)}=\delta_{2}^{(j)}$ & 0.001 & 0.488 & 0.624 & \\
\hline \multirow{3}{*}{ FR-US } & upper-tail parameter & 0.626 & 2.765 & & 0.001 \\
\hline & lower-tail parameter & 0.912 & 2.371 & & 0.015 \\
\hline & $p$-value for $\delta_{1}^{(j)}=\delta_{2}^{(j)}$ & 0.202 & 0.661 & & \\
\hline \multirow{3}{*}{ GE-UK } & upper-tail parameter & 0.340 & 8.055 & & 0.000 \\
\hline & lower-tail parameter & 0.420 & 3.640 & & 0.000 \\
\hline & $p$-value for $\delta_{1}^{(j)}=\delta_{2}^{(j)}$ & 0.713 & 0.000 & & \\
\hline \multirow{3}{*}{ GE-US } & upper-tail parameter & 0.000 & 3.393 & & 0.000 \\
\hline & lower-tail parameter & 0.906 & 1.706 & & 0.000 \\
\hline & $p$-value for $\delta_{1}^{(j)}=\delta_{2}^{(j)}$ & 0.000 & 0.000 & & \\
\hline \multirow{3}{*}{ UK-US } & upper-tail parameter & 0.506 & 2.674 & & 0.000 \\
\hline & lower-tail parameter & 0.803 & 2.430 & & 0.000 \\
\hline & $p$-value for $\delta_{1}^{(j)}=\delta_{2}^{(j)}$ & 0.007 & 0.493 & & \\
\hline
\end{tabular}

Note: The table reports the estimates of the SHR copula parameters for each regime and the $p$-values of the two hypothesis tests. The first test is about equivalence of the upper and lower tail copula parameters between the first and last regimes. The second test is about equivalence between the upper and lower tail copula parameters across each regime. The results are based on international stock returns in US dollars. 
Table 3: Results of hypothesis testing (local currency)

\begin{tabular}{|c|c|c|c|c|c|}
\hline & & $\delta_{i}^{(1)}$ & $\delta_{i}^{(2)}$ & $\delta_{i}^{(3)}$ & $\begin{array}{c}p \text {-value for } \delta_{i}^{(1)}=\delta_{i}^{(2)} \text { or } \\
\delta_{i}^{(1)}=\delta_{i}^{(3)}\end{array}$ \\
\hline \multirow{3}{*}{ FR-GE } & upper-tail parameter & 0.000 & 1.928 & 4.633 & 0.000 \\
\hline & lower-tail parameter & 0.945 & 1.375 & 4.384 & 0.000 \\
\hline & $p$-value for $\delta_{1}^{(j)}=\delta_{2}^{(j)}$ & 0.000 & 0.048 & 0.849 & \\
\hline \multirow{3}{*}{ FR-UK } & upper-tail parameter & 0.865 & 2.105 & 3.990 & 0.000 \\
\hline & lower-tail parameter & 0.692 & 1.480 & 3.612 & 0.000 \\
\hline & $p$-value for $\delta_{1}^{(j)}=\delta_{2}^{(j)}$ & 0.487 & 0.084 & 0.695 & \\
\hline \multirow{3}{*}{ FR-US } & upper-tail parameter & 0.537 & 3.031 & & 0.018 \\
\hline & lower-tail parameter & 0.924 & 2.925 & & 0.013 \\
\hline & $p$-value for $\delta_{1}^{(j)}=\delta_{2}^{(j)}$ & 0.087 & 0.859 & & \\
\hline \multirow{3}{*}{ GE-UK } & upper-tail parameter & 0.000 & 5.450 & & 0.000 \\
\hline & lower-tail parameter & 0.615 & 2.809 & & 0.000 \\
\hline & $p$-value for $\delta_{1}^{(j)}=\delta_{2}^{(j)}$ & 0.000 & 0.000 & & \\
\hline \multirow{3}{*}{ GE-US } & upper-tail parameter & 0.000 & 2.727 & & 0.017 \\
\hline & lower-tail parameter & 0.885 & 1.802 & & 0.000 \\
\hline & $p$-value for $\delta_{1}^{(j)}=\delta_{2}^{(j)}$ & 0.000 & 0.000 & & \\
\hline \multirow{3}{*}{ UK-US } & upper-tail parameter & 0.389 & 2.613 & & 0.003 \\
\hline & lower-tail parameter & 0.491 & 2.212 & & 0.000 \\
\hline & $p$-value for $\delta_{1}^{(j)}=\delta_{2}^{(j)}$ & 0.509 & 0.396 & & 0.000 \\
\hline
\end{tabular}

Note: The table reports the estimates of the SHR copula parameters for each regime and the $p$-values of the two hypothesis tests. The first test is about equivalence of the upper and lower tail copula parameters between the first and last regimes. The second test is about equivalence between the upper and lower tail copula parameters across each regime. The results are based on international stock returns in local currency. 
Figure 1: Dynamics of three dependence measures (US dollars)
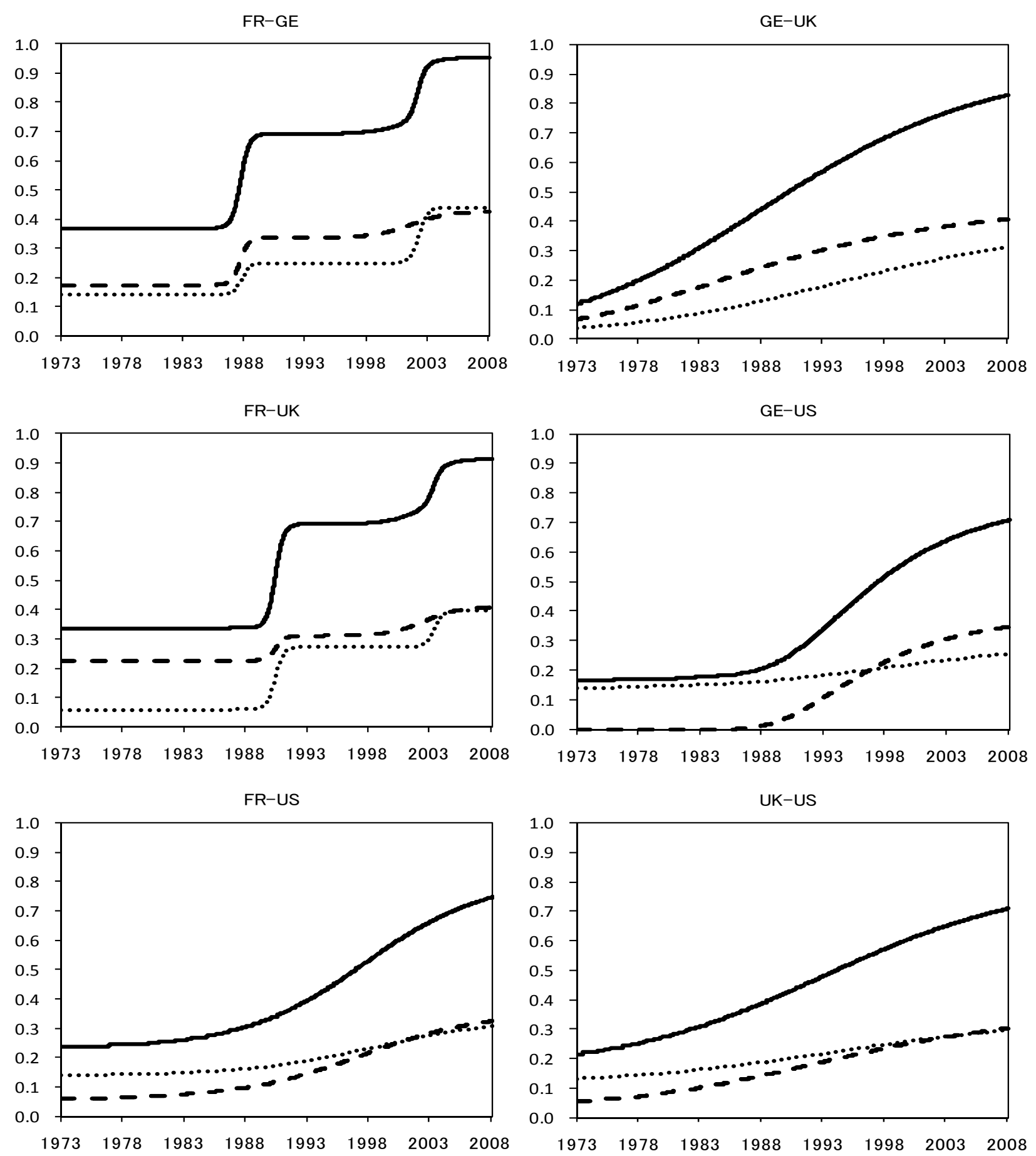

Note: The figure plots the time series of three dependence measures (Spearman's rho, upper and lower dependences) between each country pair's stock returns in US dollars implied by the best fitting smooth-transition SHRC model. The solid line shows the implied spearman's rho. The broken line shows the implied upper tail dependence. The dotted line shows the implied lower dependence. 
Figure 2: Dynamics of three dependence measures (local currency)
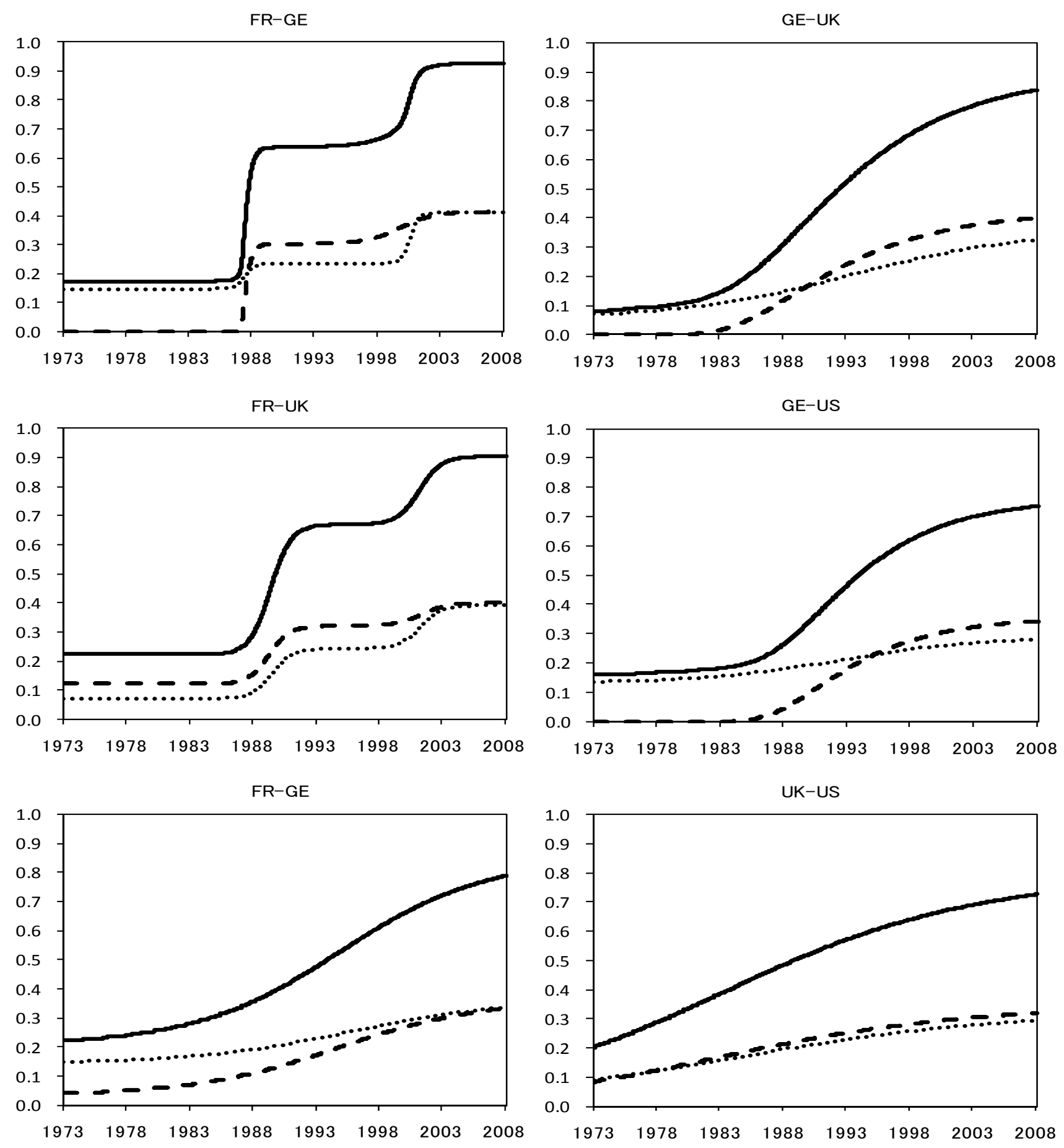

Note: The figure plots the time series of three dependence measures (Spearman's rho, upper and lower dependences) between each country pair's stock returns in local currency implied by the best fitting smooth-transition SHRC model. The solid line shows the implied spearman's rho. The broken line shows the implied upper tail dependence. The dotted line shows the implied lower dependence. 
Figure 3: Dynamics of three dependence measures (ST marginal model)
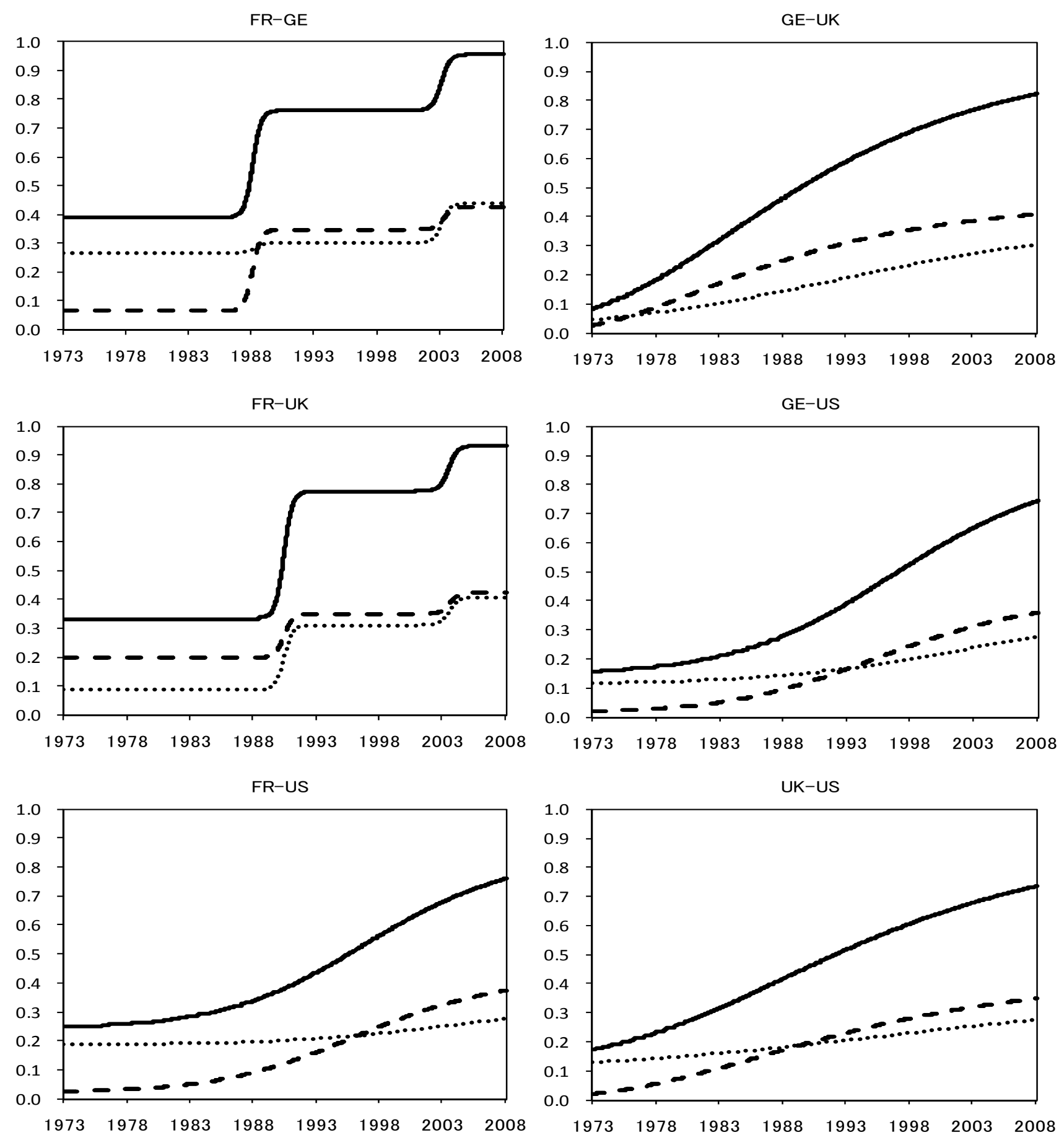

Note: The figure plots the time series of three dependence measures (Spearman's rho, upper and lower dependences) between each country pair's stock returns in US dollars implied by the best fitting smooth-transition SHRC model with the two-state smooth-transition marginal model. The solid line shows the implied spearman's rho. The broken line shows the implied upper tail dependence. The dotted line shows the implied lower dependence. 
Figure 4: Dynamics of VaR and ES ratios of $99 \%$ VaR and ES of every 5 years to those of 1973
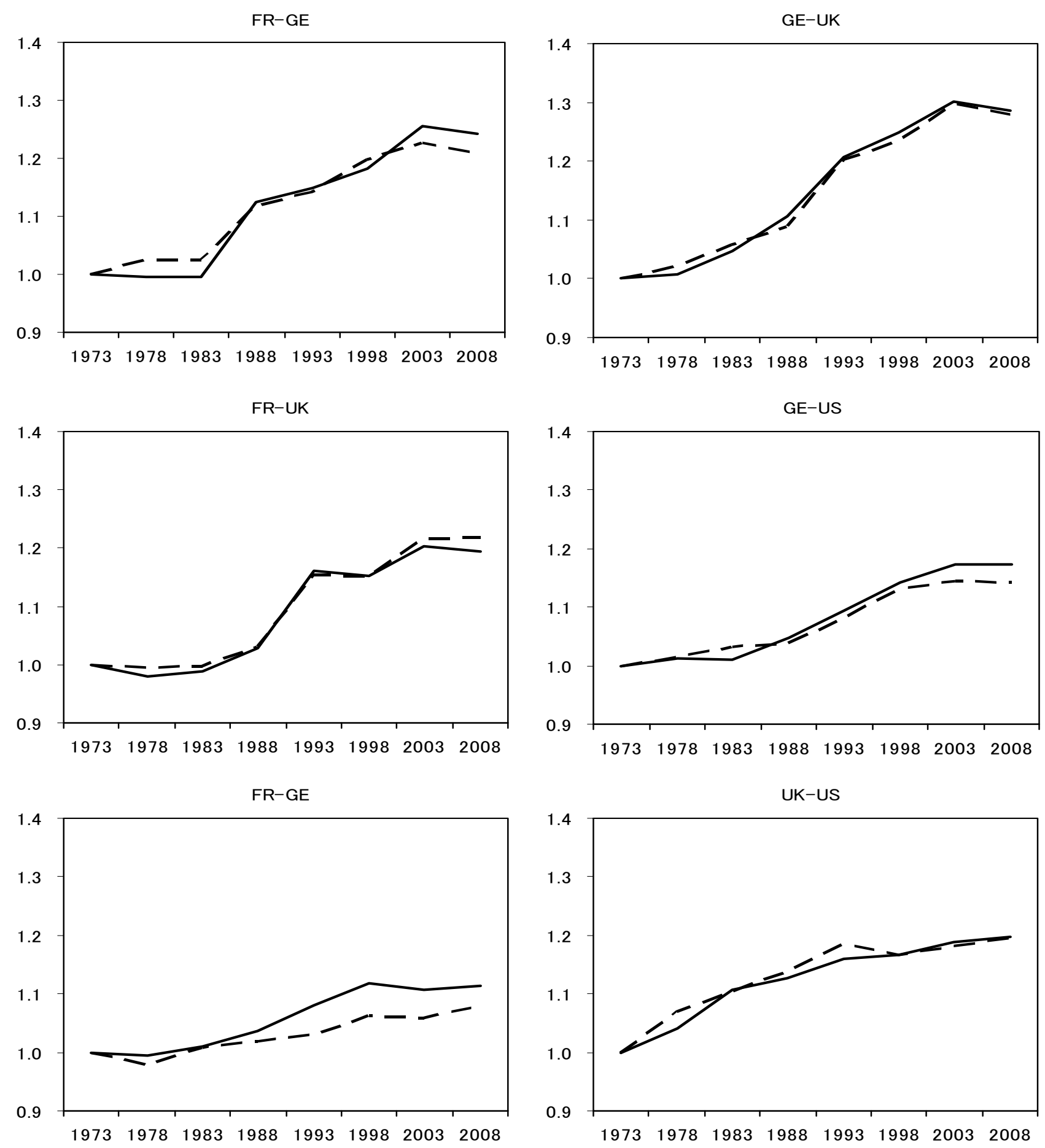

Note: The figure plots the VaR and ES ratios of $99 \%$ VaR and ES of every 5 years to those of 1973 . The calculation is based on the results from the estimated best SHRC model for international stock returns in US dollars. The solid line shows the VaR ratio. The broken line shows the ES ratio. 
Figure 5: Dynamics of VaR and ES ratios of the minimized 99\% VaR and ES to those of the less risky country
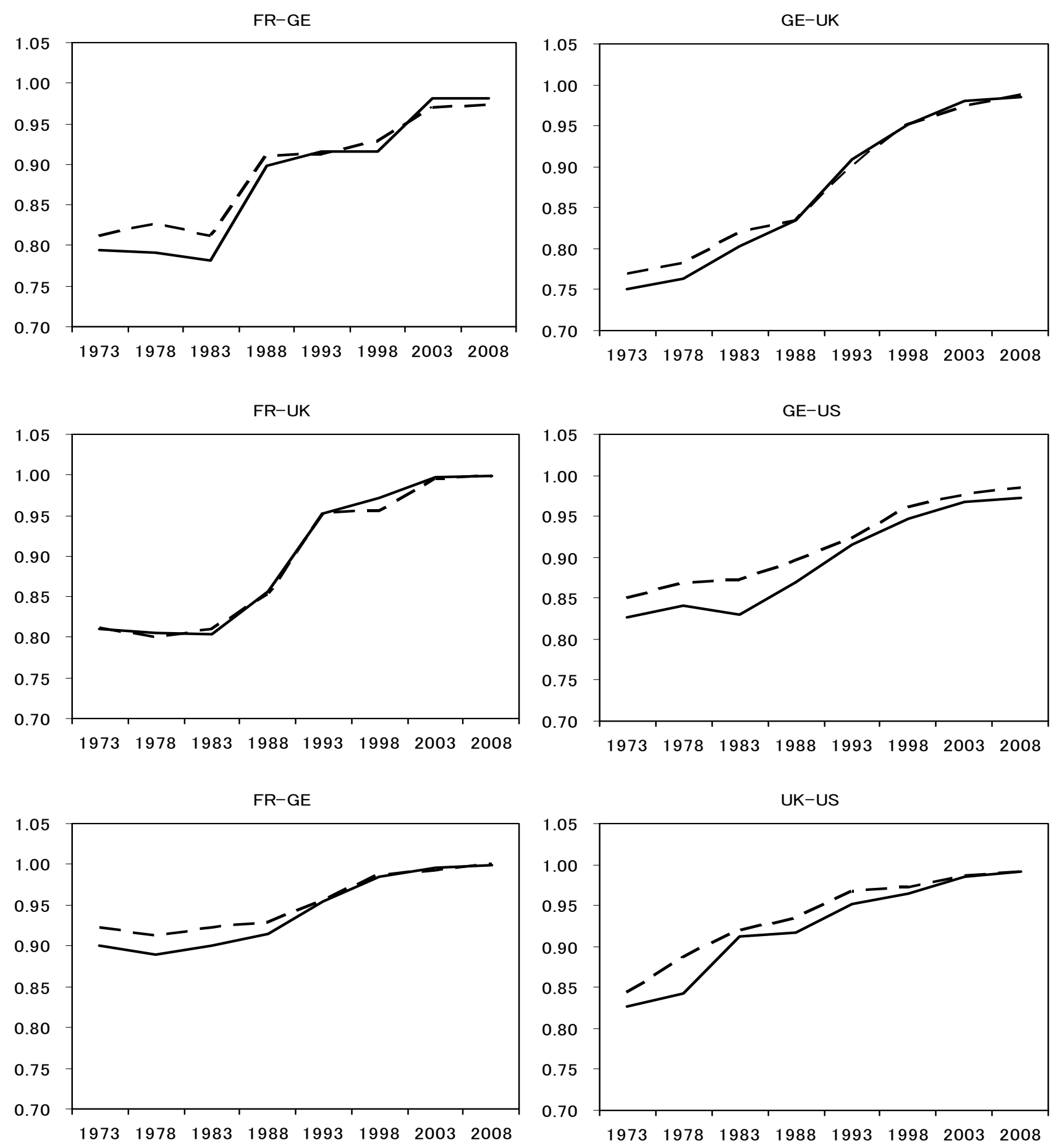

Note: The figure plots the VaR and ES ratios of the minimized $99 \%$ VaR and ES to those of the less risky country. The calculation is based on the results from the estimated best SHRC model for international stock returns in US dollars. The solid line shows the VaR ratio. The broken line shows the ES ratio. 